07,03

\title{
Образование дислокационных пар в гетероструктуре $\mathrm{Ge} / \mathrm{GeSi} / \mathrm{Si}(001)$
}

\author{
( Ю.Б. Болховитянов, А.К. Гутаковский, А.И. Дерябин, Л.В. Соколов
}

Институт фризики полупроводников им. А.В. Ржанова РАН,

Новосибирск, Россия

ฯ E-mail: sokolov@isp.nsc.ru

(Поступила в Редакцию 21 мая 2018 г.

В окончательньй редакции 20 августа 2018 г.)

В гетероструктурах (ГС) Ge/LTGe/GeSi/Si $(001)$ буферный слой $\mathrm{GeSi}$ в определенном диапазоне параметров ГС и режимов их роста остается псевдоморфным, в то время как пленка Ge полностью релаксирована за счет сетки краевых дислокаций, расположенной на границе раздела $\mathrm{Ge} / \mathrm{GeSi}$. В работе экспериментально показано, что наряду с краевыми дислокациями образуются дислокации с векторами Бюргерса типа $a_{0}\langle 100\rangle$. Их формирование обусловлено реакцией $60^{\circ}$-х дислокаций, имеющих однонаправленную винтовую компоненту. При этом, если в процессе релаксации буферного слоя краевые дислокации расщеплялись с образованием дислокационного комплекса краевого типа, в котором $60^{\circ}$-е дислокации оставались связанными, то дислокации с векторами Бюргерса $a_{0}\langle 001\rangle$ расщеплялись на две независимые $60^{\circ}$-е дислокации.

Электронно-микроскопические исследования проводились на оборудовании ЦКП „Наноструктуры“ при поддержке Российского научного фонда (грант № 14-22-00143).

DOI: 10.21883/FTT.2019.02.47127.139

\section{1. Введение}

Краевые дислокации Ломера являются одним из основных структурных дефектов в кристаллах и пленках $\mathrm{Ge}, \mathrm{Si}$ и $\mathrm{A}^{\mathrm{III}} \mathrm{B}^{\mathrm{V}}[1,2]$. В гетероструктурах $\mathrm{Ge} / \mathrm{Si}(001)$ они являются основным типом дислокаций несоответствия (ДН), компенсирующих разность параметров кристаллической решетки пленки и подложки. Дислокации Ломера образуются вследствие объединения двух комплементарных $60^{\circ}$-х ДН либо при их случайной встрече, либо в процессе наведенного зарождения [3]. Объединение двух комплементарных $60^{\circ}$-х ДН в одну краевую ДН - энергетически выгодный процесс, так как при этом взаимно компенсируются противоположно направленные винтовые компоненты вектора Бюргерса $60^{\circ}$-х ДН, не участвующие в процессе пластической релаксации напряженной пленки. Критерий $b^{2}$ и расчеты удельной энергии дислокации на атомном уровне показывают значительный энергетический выигрыш при образовании краевой дислокации [4].

Дислокации Ломера, обычно обнаруживаемые в области границ раздела, могут иметь разнообразную структуру ядра: от компактной, подобной предложенной Hornsta [5], до рыхлой, занимающей в поперечнике несколько nm [6]. Как ранее было показано [7], параметры гетероструктур (ГС) Ge/LTGe/GeSi/Si(001) и режимы их роста могут быть выбраны так, что буферный слой $\mathrm{GeSi}$ остается псевдоморфным и пленка Ge зарождается на искусственной подложке $\mathrm{GeSi} / \mathrm{Si}(001)$, латеральный параметр решетки которой повторяет латеральный параметр Si. В ГС такого типа после пластической релаксации слоя $\mathrm{Ge}$, выращенного при $500^{\circ} \mathrm{C}$, практически все дислокации несоответствия имеют краевой тип и расположены в границе раздела $\mathrm{Ge} / \mathrm{GeSi}$, а буферный слой $\mathrm{GeSi}$ остается псевдоморфным (рис. 1,a). При отжиге ГС часть ДН краевого типа, ранее считавшиеся неподвижными, мигрируют с верхней границы $\mathrm{Ge} / \mathrm{GeSi}$ на нижнюю $\mathrm{GeSi} / \mathrm{Si}$ (рис. 1, b). Такое перераспределение краевых дислокаций между верхней и нижней границами осуществляется путем движения дислокационных комплексов ломеровского типа, имеющих более рыхлую, чем компактная по Hornstra [5] краевая дислокация, атомную структуру, то есть, миграция краевых дислокаций происходит в расщепленном виде. Предполагаемый механизм их передвижения был предложен в [8]. В [9] было установлено, что расщепление затрагивает не

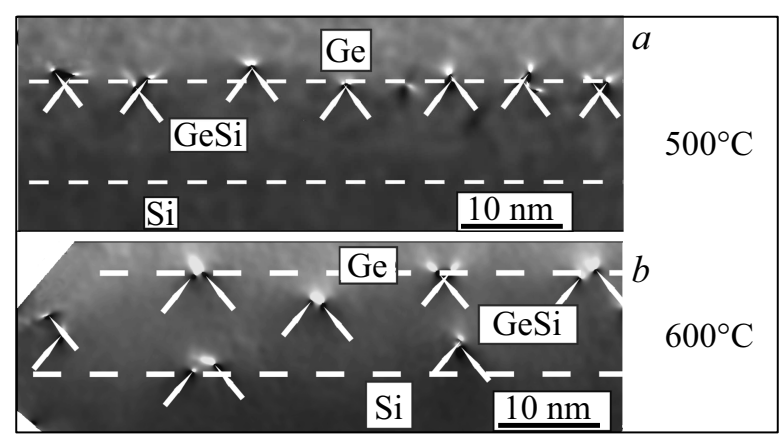

Рис. 1. Результаты цифровой обработки ВРЭМ изображений свежевыращенного при $500^{\circ} \mathrm{C}(a)$ и отожженного при $600^{\circ} \mathrm{C}$ (b) образцов гетероструктуры $\mathrm{Ge} / \mathrm{Ge}_{0.5} \mathrm{Si}_{0.5}(10 \mathrm{~nm}) /$ $\mathrm{Si}(001)$. Представлены карты распределения межплоскостных расстояний для (111) и (11) пл) плоскостей в тоновой моде (использован GPA анализ для отображения общей картины распределения дислокаций). Белые стрелки отмечают окончания экстра плоскостей $\{111\}$. Штриховые линии - границы раздела. 
только дислокации Ломера с исходно рыхлой структурой ядра, но и совершенные (компактные) дислокации Ломера.

Несмотря на преобладание дислокаций Ломера и ломеровских комплексов в границах раздела релаксированных гетероструктур, в литературе обсуждается возможность образования дислокаций с векторами Бюргерса типа $a_{0}\langle 001\rangle[10]$. Такие дислокации гипотетически могут сформироваться в результате реакции двух $60^{\circ}$-х дислокаций, имеющих однонаправленную винтовую компоненту. Oktyabrsky и J. Narayan [11], рассчитав взаимодействие дислокационных пар в разной парной комбинации, показали, что в обеих парах $60^{\circ}$-х дислокаций (с параллельными и антипараллельными винтовыми компонентами) наблюдается взаимное притяжение, однако, притяжение в паре с параллельными винтовыми компонентами в 10 раз слабее. Тем не менее, достоверных наблюдений таких дислокаций в литературе не встречается. Учитывая высокую плотность дислокаций, хорошую упорядоченность дислокационной сетки и возможность управления их перемещением, можно ожидать наблюдения дислокаций типа $a_{0}\langle 001\rangle$ в гетероструктуре $\mathrm{Ge} / \mathrm{LTGe} / \mathrm{GeSi} / \mathrm{Si}(001)$.

\section{2. Эксперимент}

Структуры Ge/LTGe/GeSi/Si(001) были выращены методом молекулярной эпитаксии. На поверхности $\mathrm{Si}$ подложки при $T=450^{\circ} \mathrm{C}$ вначале выращивался $10 \mathrm{~nm}$ буферный слой $\mathrm{GeSi}$ с содержанием $\mathrm{Ge}$, равным 0.5; затем, при $T=200^{\circ} \mathrm{C}$, наносился затравочный слой $\mathrm{Ge}(\mathrm{LT} \mathrm{Ge})$ толщиной $7 \mathrm{~nm}$. После этого, одновременно с подьемом температуры до $T=500^{\circ} \mathrm{C}$, выращивался основной слой $\mathrm{Ge}$ до толщины $0.5 \mu \mathrm{m}$. По окончании роста образцы отжигались в атмосфере водорода при температуре $600^{\circ} \mathrm{C}$ в течение $10 \mathrm{~min}$.

Дислокационная структура выращенных образцов изучалась на поперечных срезах на электронном микроскопе JEOL-4000EX с ускоряющим напряжением $400 \mathrm{kV}$. Использовалась высокоразрешающая электронная микроскопия (ВРЭМ), сканирующая просвечивающая электронная микроскопия (СПЭМ), а также цифровая обработка ВРЭМ и СПЭМ изображений [12].

$\mathrm{B}$ настоящей работе использовалась методика анализа геометрической фазы (GPA - geometric phase analysis), впервые предложенная в [13], для изучения локального распределения межплоскостных расстояний $d_{111}$ и $d_{1 \overline{1} 1}$ в окрестности дислокаций несоответствия. Детали методики можно найти в [14]. Цифровая обработка экспериментальных ВРЭМ и СПЭМ изображений осуществлялась с использованием коммерческой программы „Digital Micrograph“ (GATAN).

\section{3. Результаты}

Как ранее было показано $[7,8,15,16]$, при отжиге дислокационные дефекты ломеровского типа перерас-

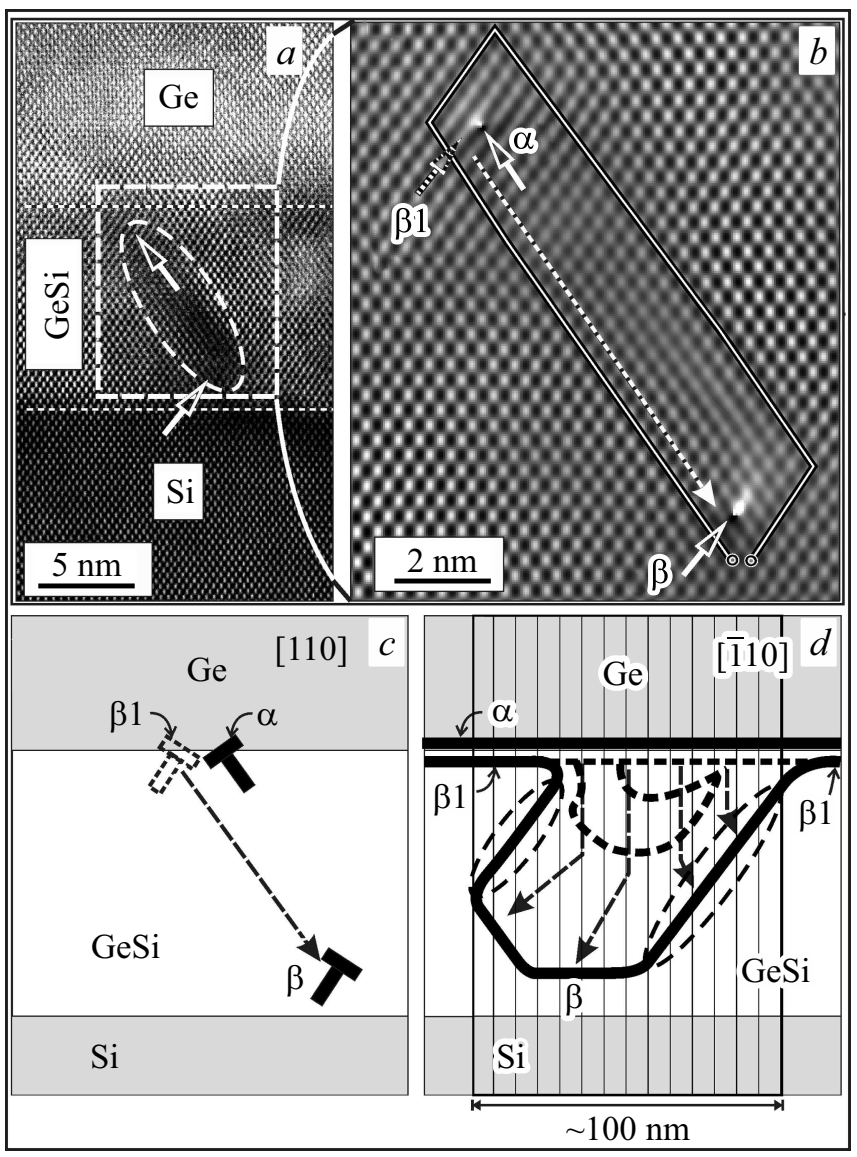

Рис. 2. (a) - экспериментальное СПЭМ-изображение поперечного среза неотожженного образца $\mathrm{Ge} / \mathrm{Ge}_{0.5} \mathrm{Si}_{0.5}(10 \mathrm{~nm}) /$ $\mathrm{Si}(001)$. Белым штриховым овалом отмечен деформационный контраст от дислокации, лежащей внутри фольги в наклонной плоскости $(111) ;(b)-$ фильтрованное в рефлексах $(11 \overline{1})$ и (111) композитное изображение области, выделенной белым прямоугольником на рис. 2, $a$. Белой пунктирной стрелкой отмечено направление возможного скольжения дислокации $\beta$ из вероятного положения $\beta 1$ в наблюдаемое положение $\beta$. $c, d-$ схемы расщепления пары $60^{\circ}$-х дислокаций в предположении их слабой связи. $(c)-$ тот же вид, что и на рис. $2, a$, $(d)$ - вид сбоку, в направлении [110].

пределяются на двух гетерограницах таким образом, что происходит компенсация несоответствия буферного слоя. При этом, в процессе перемещения части дефектов с границы $\mathrm{Ge} / \mathrm{GeSi}$ в границу $\mathrm{GeSi} / \mathrm{Si}$ происходит уменьшение доли дислокаций с компактным ядром в пользу „рыхлых“ дислокаций, обладающих ядром в $2-6 \mathrm{~nm}[8,15,16]$. Несмотря на увеличение расстояния между $60^{\circ}$-ми дислокациями, образующими краевую дислокацию, в целом, большинство $60^{\circ}$-х дислокаций не теряют связи, а образуют рыхлый дефект, перемещающийся как единое целое, по механизму, описанному в [16].

Тем не менее встречаются и не связанные друг с другом, на первый взгляд, $60^{\circ}$-е дислокации. Один из таких примеров представлен на рис. 2. 
Внимательное рассмотрение позволяет обнаружить деформационный контраст между парой $60^{\circ}$-х дислокаций (выделено белым штриховым овалом на рис. 2,a). В литературе встречаются предположения о возможности существования на границе раздела пленка/подложка парных $60^{\circ}$-х дислокаций с параллельными винтовыми компонентами, например, в системе $\mathrm{Ge} / \mathrm{Si}[6,17]$, в противоположность комплементарным дислокациям, у которых винтовые компоненты антипараллельны. Такие пары не имеют энергетических преференций перед раздельными $60^{\circ}$-ми дислокациями однако они также выполняют основную функцию дислокаций несоответствия в ГС релаксацию напряжений в пленке. Не будучи сильно связанной, такая дислокационная пара может легко распасться на две независимые $60^{\circ}$-е дислокации. Предположительная конфигурация $60^{\circ}$-х дислокаций, наблюдающихся на рис. $2, a, 2 b$ представлена на рис. 2, c. Здесь изображена пара $60^{\circ}$-х дислокаций $(\alpha$ и $\beta)$, находящихся на наклонных зеркально отклоненных плоскостях $\{111\}$. Одна из них $(\alpha)$ находится вблизи границы $\mathrm{Ge} / \mathrm{GeSi}$, тогда как участок дислокации $\beta$ проскользил вдоль плоскости (111) почти до границы GeSi/Si. Деформационный контраст, наблюдаемый на рис. 2, a (осевая линия деформационного следа совпадает со следом наклонной плоскости (111)), создается наклонным участком дислокации $\beta$ (выделен белым штриховым овалом на рис. $2, d$ ). Если дислокацию $\beta$ вернуть вдоль плоскости скольжения (111) в положение $\beta 1$, получится пара близко расположенных $60^{\circ}$-х дислокаций. Схема расщепления (предполагаемый вид в направлении, лежащем в плоскости фольги) представлена на рис. 2, $d$. На первоначально прямой линии дислокационной пары $60^{\circ}$-х дислокаций $(\alpha$ и $\beta 1)$ образуется полупетля (дислокация $\beta$ ), пересекающая буферный слой GeSi. Штриховыми овалами на рис. 2, $d$ выделены наклонные участки $60^{\circ}$-й дислокации, ответственные за появление деформационного контраста, видимого на рис. 2, a. Конечная дислокационная полупетля на рис. $2, d$ по форме скопирована с наблюдавшейся ранее экспериментально в работе [18]. В отличие от наблюдающихся в массовом порядке рыхлых ломеровских комплексов краевого типа, мы обнаружили только одну пару связанных деформационным контрастом $60^{\circ}$-х дислокаций. Дополнительным свидетельством в пользу слабой связи этих дислокаций является их высокая подвижность при температуре роста. Если Ломеровские комплексы обнаруживают движение к границе $\mathrm{Ge} / \mathrm{GeSi}$ только после отжига, то в рассматриваемой паре $60^{\circ}$-х дислокаций одна из них уже проскользила к границе $\mathrm{GeSi} / \mathrm{Si}$ во время роста. Необходимо отметить, что в случае параллельных винтовых компонент у пары $60^{\circ}$-х дислокаций, контур Бюргерса на рис. $2, b$ не является плоским четырехугольником, так что вектор Бюргерса, замыкающий этот контур, имеет компоненту, направленную перпендикулярно плоскости рисунка.

\section{4. Обсуждение результатов}

В 1951 г.Ломер [1] предположил, что упрочнение кубических и гексагональных кристаллов при деформации

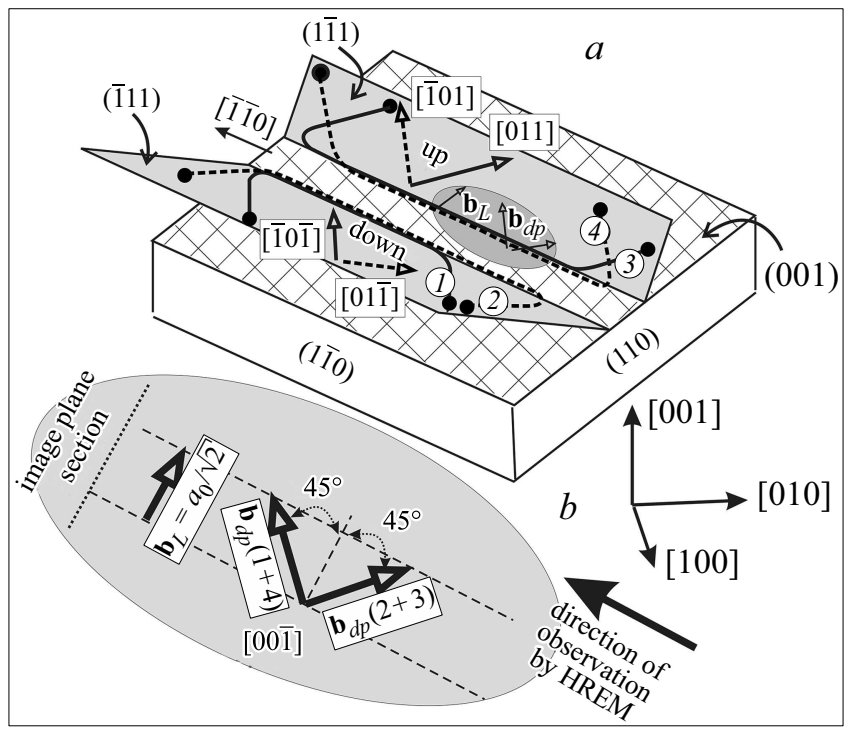

Рис. 3. Схемы движения краевых дислокаций Ломера, а также $60^{\circ}$-х дислокационных пар в гетероструктурах $\mathrm{Ge} / \mathrm{GeSi} / \mathrm{Si}(001)$ (a) - четыре семейства $60^{\circ}$-х дислокаций типа $a / 2\langle 110\rangle\{111\}$, участвующих в пластической релаксации напряженной пленки $\mathrm{GeSi}$ на $\mathrm{Si}$ и распространяющихся в одном из направлений $\langle 110\rangle$. Граница раздела пленка/подложка заштрихована. Направления векторов Бюргерса взяты из работы [19]. (b) - вид сверху (направление [001] ) области, ограниченной на $(a)$ овалом. $\mathbf{b}_{d p}-$ суммарные векторы Бюргерса дислокационных пар (1)+(4) и (2)+(3).

может быть объяснено появлением краевых сидячих дислокаций. В гетеросистеме $\mathrm{Ge}$ на $\mathrm{Si}$ (001) эти дислокации (названные дислокациями Ломера) образуются из двух $60^{\circ}$-х дислокаций, скользящих вдоль двух зеркально наклонных плоскостей $\{111\}$, пересекающихся в границе раздела пленка/подложка. В результате их взаимодействия образуется краевая дислокация. Применительно к системе $\mathrm{GeSi} / \mathrm{Si}(001)$ в каждом из двух ортогональных направлений $\langle 110\rangle$ распространяются четыре семейства $60^{\circ}$-х дислокаций - по две на каждой зеркально наклонной плоскости скольжения $\{111\}$. На рис. 3, a эти пары обозначены как (1), (2) (плоскость (111)) и (3), (4) (плоскость (111)). Краевая дислокация Ломера образуется при взаимодействиях (1)+(3) и (2)+(4) дислокаций по следующим выражениям:

$$
\begin{aligned}
& \mathbf{b}_{1}+\mathbf{b}_{3}=a_{0} / 2[\overline{1} 0 \overline{1}]+a_{0} / 2[011]=a_{0} / 2[\overline{1} 10], \\
& \mathbf{b}_{2}+\mathbf{b}_{4}=a_{0} / 2[01 \overline{1}]+a_{0} / 2[\overline{1} 01]=a_{0} / 2[\overline{1} 10],
\end{aligned}
$$

где $a_{0}$ - параметр решетки.

В обоих случаях вектор Бюргерса $\mathbf{b}_{L}$ такой дислокации лежит в плоскости (001) и перпендикулярен линии дислокации (рис. $3, b$ ). В соответствии с правилом Франка [20] эта реакция энергетически выгодна: $b_{1}^{2}+b_{3}^{2}>b_{L}^{2}$, также как и $b_{2}^{2}+b_{4}^{2}>b_{L}^{2}$. Действительно, при таком взаимодействии происходит компенсация антипараллельных винтовых компонент $60^{\circ}$-х дислокаций, 
участвующих в реакции. Соответственно такие пары $60^{\circ}$-х дислокаций, называемые комплементарными [3], испытывают притяжение друг к другу и при возможности сближения объединяются в компактные краевые дислокации Ломера. Рассчитанная в [4] энергия взаимодействия комплементарных дислокаций, образующих ломеровскую составляет $\sim 27 \mathrm{eV} / \mathrm{nm}$.

Комбинации $60^{\circ}$-х дислокаций, находящихся на зеркально отклоненных плоскостях $\{111\}$, но с параллельными винтовыми компонентами (на рис. 3 это (1)+(4) и (2)+(3)), приведены ниже

$$
\begin{aligned}
& \mathbf{b}_{1}+\mathbf{b}_{4}=a_{0} / 2[\overline{1} 0 \overline{1}]+a_{0} / 2[\overline{1} 0 \overline{1}]=a_{0}[\overline{1} 00], \\
& \mathbf{b}_{2}+\mathbf{b}_{3}=a_{0} / 2[01 \overline{1}]+a_{0} / 2[001]=a_{0}[010] .
\end{aligned}
$$

Результирующий вектор Бюргерса такой реакции также лежит в плоскости (001), но направлен под углом 45 к линии дислокации (рис. $3, b$ ). Таким образом, необходимый признак краевой дислокации - перпендикулярность вектора Бюргерса к ее линии - не выполняется, и результат такого взаимодействия не является краевой дислокацией. Однако существование и присутствие таких дислокационных пар в буферном слое $\mathrm{GeSi}$ и его границах не может быть выявлено на ВРЭМ снимках поперечных сечений образцов, параллельных плоскости (110). Как видно на рис. $3, b$, проекции векторов Бюргерса $\mathbf{b}_{d p}$ на плоскость ВРЭМ или СПЭМ изображений, совпадают по величине с вектором Бюргерса ломеровской краевой дислокации $\mathbf{b}_{L}$. Поэтому невозможно выявить некомплементарные пары $60^{\circ}$-х дислокаций на фоне многочисленных ломеровских комплексов с помощью обхода контуром Бюргерса. Тем не менее, по правилу Франка $\left(b_{1}^{2}+b_{4}^{2}=b_{d p}\right.$ или $\left.b_{2}^{2}+b_{3}^{2}=b_{d p}^{2}\right)$ такое взаимодействие предполагается возможным. Так, в работе [6] авторы считают, что дислокационные пары, не сформировавшие компактную дислокацию Ломера после отжига образца, и есть пары $60^{\circ}$-х дислокаций с параллельными винтовыми компонентами, то есть, результаты выражений (дислокационных реакций) (3 и 4). В то же время в наших образцах большинство дислокационных пар имеют рыхлую структуру, в то время как вследствие механизма наведенного зарождения [21] можно ожидать, что комплементарые $60^{\circ}$-е дислокации зарождаются парами. Можно предположить, что отличие комплементарной пары $60^{\circ}$-х дислокаций от некомплементарной может быть обнаружено только по разнице взаимодействия дислокаций между собой. В случае некомплементарной пары это либо не образование компактной дислокации Ломера даже после отжига, либо как это показано на (рис. 3), расщепление дислокационного комплекса на две $60^{\circ}$-е дислокации даже при низкой температуре.

\section{5. Заключение}

Приведенные экспериментальные данные и обработка более 300 дислокационных пар свидетельствуют о преобладающей роли краевых дислокаций и дислокационных комплексов краевого типа в релаксации гетероструктуры $\mathrm{Ge} / \mathrm{Ge}_{0.5} \mathrm{Si}_{0.5} / \mathrm{Si}(001)$. Тем не менее, в малом количестве встречаются дислокационные конфигурации, состоящие из двух некомплементарных $60^{\circ}$-х дислокаций, имеющих однонаправленную винтовую компоненту. Суммарный вектор Бюргерса такого дефекта имеет значение типа $a_{0}\langle 100\rangle$.

\section{Список литературы}

[1] W.M. Lomer. Phil. Mag. 42, 1327 (1951).

[2] S. Mader, A.E. Blakeslee, J. Angilello. J. Appl. Phys. 45, 4730 (1974).

[3] E.P. Kvam, D.M. Maher, C.J. Humpreys. J. Mater. Res. 5, 1900 (1990).

[4] M. Ishimura, J. Narayan. Phil. Mag. A 72, 281 (1995).

[5] J. Hornstra. J. Phys. Chem. Solid 5, 129 (1958).

[6] J. Narayan, S. Oktyabrsky. J. Appl. Phys. 92, 7122 (2002).

[7] Yu.B. Bolkhovityanov, A.S. Deryabin, A.K. Gutakovskii, L.V. Sokolov. Acta Mater. 61, 617 (2013).

[8] Ю.Б. Болховитянов, А.К. Гутаковский, А.С. Дерябин, Л.В. Соколов. ЖЭТФ 150, 5 (11), 955 (2016).

[9] Yu.B. Bolkhovityanov, A.S. Deryabin, A.K. Gutakovskii, L.V. Sokolov. J. Crystal Growth 483, 265 (2018).

[10] Yi Wang, P. Ruterana, S. Kret, J. Chen, S. El Kazzi, L. Desplanque, X. Wallart. Appl. Phys. Lett. 100, 262110 (2012).

[11] S. Oktyabrsky, J. Narayan. MRS Proc. 399, 443 (1996).

[12] M.J. Hÿtch, E. Snoeck, R. Kilaas. Ultramicroscopy 74, 131 (1998).

[13] S. Kret, P. Ruterana, A. Rosenauer, D. Gerthsen. Phys. Status Solidi B 227, 247 (2001).

[14] А.К. Гутаковский, А.Л. Чувилин, С.А. Сонг. Доклады РАН. Физика 71, 1426 (2007). [Available at https://www.researchgate.net/publication/225617264.]

[15] Y.B. Bolkhovityanov, A.S. Deryabin, A.K. Gutakovskii, L.V. Sokolov. Acta Mater. 61, 5400 (2013).

[16] Yu.B. Bolkhovityanov, A.S. Deryabin, A.K. Gutakovskii, L.V. Sokolov. Thin Solid Films 616, 348 (2016).

[17] A. Vila, A. Cornet, J.R. Morante. Appl Phys Lett. 68, 1244 (1996).

[18] Yu.B. Bolkhovityanov, A.S. Deryabin, A.K. Gutakovskii, M.A. Revenko, L.V. Sokolov. J. Crystal Growth 293, 247 (2006).

[19] R.S. Goldman, K.L. Kavanagh, H.H. Wieder, S.N. Ehrlich, R.M. Feenstra. J. Appl. Phys. 83, 5137 (1998).

[20] F.C. Frank. Physika 15, 131 (1949).

[21] Yu.B. Bolkhovityanov, A.S. Deryabin, A.K. Gutakovskii, L.V. Sokolov. ФTT 52, 1, 32 (2010).

Редактор Ю.Э. Китаев 